\title{
DOSSIER
}

\section{Creación de espacios de aprendizaje y enseñanza para la investigación transformadora y transdisciplinar: el Laboratorio de Innovación Transformadora ${ }^{1}$ \\ Creating Learning and Teaching Spaces for Transformative and Transdisciplinary Research: the Transformative Innovation Lab}

\author{
Matthias Wanner \\ Wuppertal Institute for Climate, Environment and Energy, Alemania \\ matthias.wanner@wupperinst.org \\ Philip Bernert \\ Leuphana University Lüneburg, Alemania \\ philip.bernert@leuphana.de \\ Nele Fischer \\ Technische Universität Berlin, Alemania \\ nele.fischer@tu-berlin.de \\ Martina Schmitt \\ Wuppertal Institute for Climate, Environment and Energy, Alemania \\ martina.schmitt@wupperinst.org
}

https://doi.org/10.48102/didac.2021..78_JUL-DIC.80

8

RESUMEN

Para lograr una transición hacia el desarrollo sostenible son fundamentales la ciencia y la educación, especialmente la educación superior. Se necesitan formatos educativos para capacitar a los estudiantes en la realización de investigaciones transformadoras. Con base en la investigación transdisciplinaria y transformadora en laboratorios del mundo real y estudios del futuro, desarrollamos un módulo de aprendizaje y enseńanza integral: el Laboratorio de Innovación Transformadora (LIT). El laboratorio desarrolla cinco competencias clave y tres tipos de conocimiento necesarios para impulsar innovaciones en sostenibilidad socialmente robustas. En este artículo se presentan las principales características de este formato vivencial y reflexivo, además de un manual para facilitar el laboratorio. También se comparten y discu-

60 - Creación de espacios de aprendizaje y enseñanza para la investigación transformadora y trasdisciplinar...

Matthias Wanner, Philip Bernert, Nele Fischer, Martina Schmitt. DIDAC 78 (2021): 60-71 
ten los aprendizajes centrales de la implementación de este formato en programas de estudio existentes a partir de dos pruebas realizadas en dos universidades alemanas.

Palabras clave: Educación para el desarrollo sostenible; transdisciplinariedad; investigación transformadora; laboratorios del mundo real; estudios del futuro; competencias clave para la sostenibilidad.

\section{ABstract}

For achieving a transition towards sustainable development, central importance is attached to science and education, and especially higher education. Suitable formats are needed for empowering students to perform transformative research. Based on transdisciplinary and transformative realworld laboratory research and futures studies, we develop encompassing learning and teaching module: the Transformative Innovation Lab. The lab builds on insights into five key competencies and three types of knowledge needed for developing socially robust sustainability innovations. In this paper, the main features of this experiential and reflexive format are presented and linked to a handbook for facilitating the lab. Central learnings for implementing the format in existing study programmes from two test runs at two German universities are shared and discussed.

Keywords: Education for Sustainable Development; Transdisciplinarity; Transformative Research; Real-World Laboratories; Futures Studies; Key Competencies for Sustainability.

Fecha de recepción: 26/02/2021

Fecha de aceptación: 16/04/2021

\section{Introducción}

A pesar de los avances globales para lograr una transición hacia un mundo "donde todas las formas de vida puedan prosperar" (UN, 2015c), muchos problemas socioambientales como la pobreza, la desigualdad o el cambio climático siguen siendo apremiantes - y en muchos casos, en proceso de rápida agudización (Steffen, Richardson et al., 2015; Steffen, Broadgate et al., 2015) —. En este contexto, la Asamblea General adoptó en 2015 la Agenda 2030 para el Desarrollo Sostenible, un plan para mejorar considerablemente la calidad de vida de la población actual y futura y proteger los recursos naturales a nivel global (onu, 2015b). Para el cumplimiento de la agenda resultan fundamentales la ciencia y la educación, en especial la educación superior con sus formatos de enseñanza y aprendizaje universitarios (UN, 2015a, 2015b; wbGU, 2011). La comprensión de la complejidad, las incertidumbres, los riesgos y el balance entre beneficios y perjuicios asociados a los retos de la sostenibilidad local y mundial se considera crucial para fomentar el desarrollo sostenible (Leicht et al., 2018). Por lo tanto, la Educación para el Desarrollo Sostenible (EDs) se centra en el desarrollo de las competencias clave que permiten a los individuos conformar y orientar los procesos de desarrollo social en una dirección sostenible.

En el ámbito científico, el Consejo Consultivo Científico del Gobierno Federal Alemán sobre el Cambio Global (wgBu, por sus siglas en ingles) ha destacado la necesidad de enfoques de investigación con orientación normativa y hacia la acción (2011). En este orden de ideas, propone que el objetivo de la investigación sobre la transformación es entender tanto los factores causales e impulsores como los obstáculos de los procesos de transformación para inferir conclusiones sobre futuros desarrollos y cómo configurarlos. Por otro lado, el objetivo de la investigación transformadora es probar soluciones de sostenibilidad en intervenciones en el mundo real y aprender durante su implementación (WBGU, 2011, pp. 321 y ss.).

En esta línea de pensamiento, tres tipos de conocimiento resultan fundamentales para comprender y diseñar transformaciones sostenibles: a) conocimiento de sistemas, $b$ ) conocimiento de objetivos y c) conocimiento de la transformación (Hirsch Hadorn et al., 2006). El conocimiento de sistemas provee una comprensión "objetiva" de las relaciones 
sistémicas. El conocimiento de objetivos explora futuros deseables y, por tanto, normativos. La investigación sobre estos dos tipos de conocimiento está vinculada a la investigación sobre la transformación y suele ser descriptiva y expositiva. El tercer tipo de conocimiento, el de la transformación, constituye el territorio de la investigación transformadora; donde - a través de procesos colaborativos y experimentales- se prueban, desarrollan y aplican conocimientos sobre las posibilidades de dar forma a procesos de transformación que sean efectivos en términos de sostenibilidad; conocimientos específicos del contexto y de los actores involucrados.

Para desarrollar estos conocimientos y ser capaz de utilizarlos se requiere una formación especial. Por lo tanto, surge la pregunta de cómo formar a los estudiantes para que realicen investigaciones transformadoras y se conviertan en agentes de cambio en materia de sostenibilidad.

En este artículo se propone un módulo de aprendizaje paso a paso que ayude a los estudiantes a descubrir su potencial para realizar investigación transformadora. Primero, mencionaremos los fundamentos conceptuales del módulo y describiremos el estado del arte de la investigación transformadora, transdisciplinaria y de los laboratorios del mundo real, así como de los estudios del futuro. Luego, nos basaremos en un marco de competencias clave para dar forma a la sostenibilidad. A partir de ahí se deriva el formato del Laboratorio de Innovación Transformadora (LIT), el cual presentaremos. Después, expondremos la implementación y los resultados de dos ejecuciones de prueba del formato. Por último, concluiremos con una discusión crítica y preguntas abiertas sobre la enseñanza de la investigación transformadora y transdisciplinaria en la educación superior.

\section{Enfoques de investigación transformadores $y$ transdisciplinarios}

La cuestión de cómo puede contribuir la ciencia a superar los retos globales descritos es un tema primordial de la investigación en sostenibilidad y ha dado lugar a un profundo proceso de reflexión den- tro de la comunidad científica, especialmente desde la década de 1990.

A partir de modelos de investigación-acción, diseños de investigación orientados a soluciones e intervenciones han sido discutidos y desarrollados en lo que es conocido como el Modo-2 de la investigación científica (Gibbons et al., 1994; Nowotny et al., 2001; Schneidewind et al., 2016). La investigación transdisciplinaria (ITD) se ha desarrollado como un modo fructífero para abordar problemas de sostenibilidad (véanse Hirsch Hadorn et al., 2008; Lang et al., 2012; Scholz \& Steiner, 2015; Thompson Klein, 2004), la cual engloba tres características esenciales:

- Aborda problemas relevantes para la sociedad.

- Permite procesos de aprendizaje compartidos e integra conocimiento de científicos de distintas disciplinas y también y particularmente de actores fuera de la comunidad científica.

- Tiene como objetivo la producción de conocimientos socialmente sólidos y orientados a soluciones que puedan transferirse tanto a la sociedad como a la ciencia.

El modelo típico-ideal de ITD basado en Lang et al. (2012) propone tres fases de investigación. La primera fase sirve principalmente para elaborar una descripción compartida del problema para abordar y conformar un equipo adecuado para el proyecto. El objetivo de la segunda fase es integrar diferentes corpus de conocimiento para cocrear un conocimiento transferible y orientado a las soluciones. La última fase consiste en reintegrar y aplicar los conocimientos incorporados en el proceso de investigación. Esta reintegración bidimensional devuelve los conocimientos a la comunidad científica y a la práctica social.

\section{Los laboratorios del mundo real como escenarios para la investigación transdisciplinar y transformadora de la sostenibilidad}

En los últimos años se han consolidado nuevos enfoques de investigación experimental y contextualizada

62 - Creación de espacios de aprendizaje y enseñanza para la investigación transformadora y trasdisciplinar... Matthias Wanner, Philip Bernert, Nele Fischer, Martina Schmitt. DIDAC 78 (2021): 60-71 
que buscan impulsar la innovación para el desarrollo sostenible (Berkhout et al., 2010; Bulkeley \& Castán Broto, 2013; Fuenfschilling et al., 2019; Nevens et al., 2013). La integración de la experimentación en el mundo real como parte constituyente de laboratorios del mundo real es la variante más elaborada de estos enfoques cuando se trata de adoptar la historia y las cualidades de la ITD (Bergmann et al., 2021; Schäpke et al., 2018; Wanner et al., 2018).
Los laboratorios del mundo real vinculan sistemáticamente la investigación transdisciplinaria y de transformación, a su vez, marcan la intersección entre la investigación de la transformación y la investigación transformadora. Éstos se enfocan en la experimentación, es decir, en la aplicación de los conocimientos cocreados y en el aprendizaje cíclico a partir de esos conocimientos en el transcurso del proceso de investigación (véase Figura 1).

\section{Figura 1}

Concepto de un proceso de laboratorio del mundo real transdisciplinario y transformador centrado en el aprendizaje a partir de la experimentación

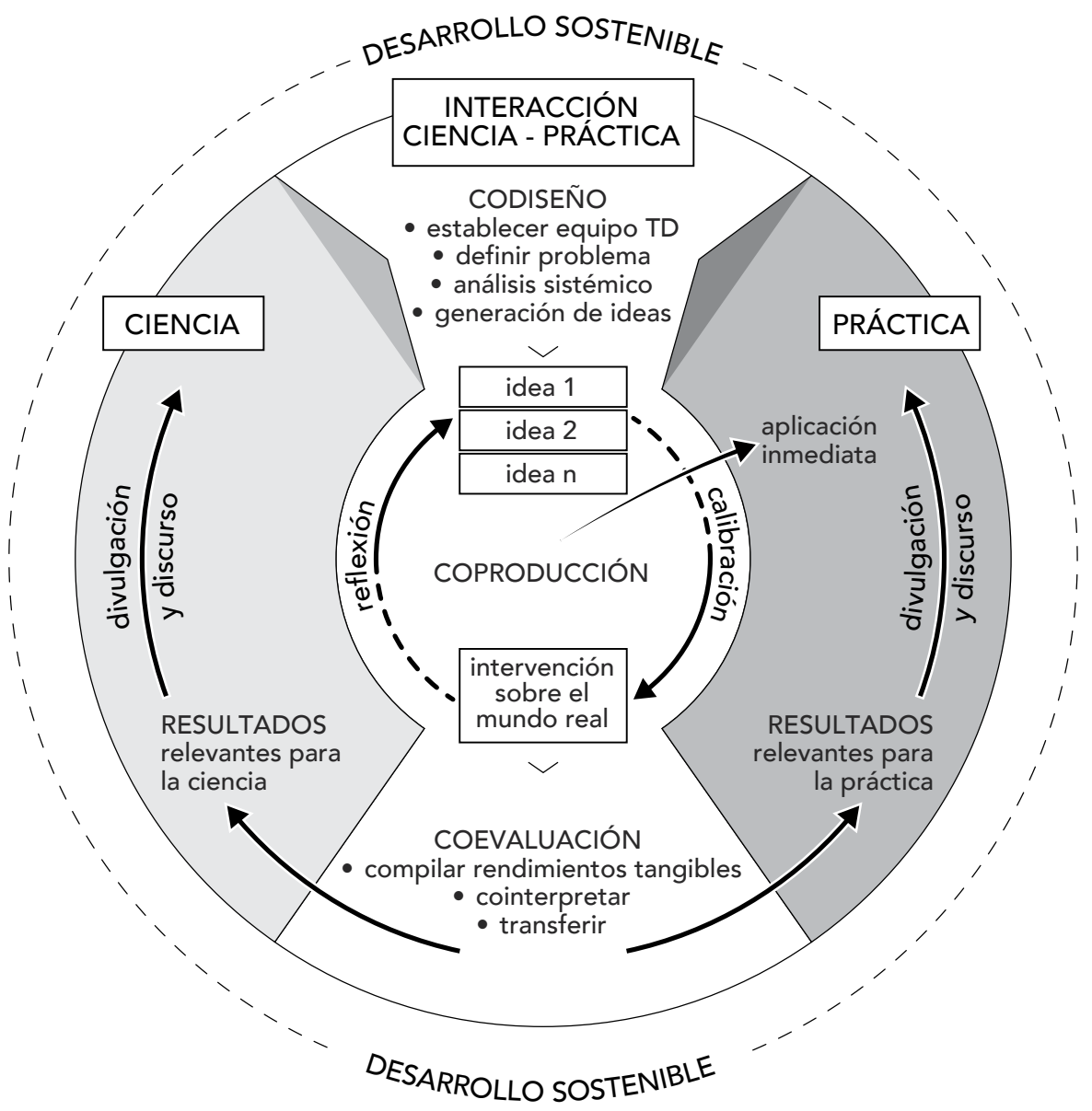

Adaptado de "Towards a Cyclical Concept of Realworld Laboratories: A Transdisciplinary Research Practice for Sustainability Transitions", por M. Wanner et al., 2018, disP - The Planning Review, 54(2), p. 102.

La figura muestra el proceso típico ideal de un laboratorio del mundo real con las fases de codiseño, coproducción y coevaluación. Esta lógica guio la conceptualización de las fases de aprendizaje en el LIT.
En este proceso, los laboratorios del mundo real siguen un enfoque de colaboración transdisciplinar entre científicos y especialistas (locales), en el que integran distintas formas de conocimiento y proyec- 
tos de participación y aprendizaje organizados conjuntamente, incluyendo formas de aprendizaje exploratorio. La investigación en los laboratorios del mundo real sucede principalmente dentro del contexto. El proceso también permite la autorreflexión y la evaluación continua para cuestionar constantemente los procesos de investigación, adaptarse a las nuevas condiciones e impulsar innovaciones. Por lo tanto, los laboratorios del mundo real son escenarios educativos para la investigación transdisciplinaria en sostenibilidad orientada a la transformación. En este sentido, amplían el enfoque habitual anterior de los procesos de ITD — que se centra en integrar y sintetizar conocimientos para su posible aplicación-.

\section{Estudios del futuro: un área de investigación relacionada}

El campo de estudios del futuro tiene como objetivo crear un conocimiento orientativo, estrechamente vinculado con el conocimiento de objetivos y de la transformación (Grunwald, 2014), así como al Modo-2 de la investigación científica. Los estudios del futuro consideran múltiples futuros posibles, e invitan a la discusión sobre posibles incertidumbres y sorpresas. Éstos también abren otras posibilidades de toma de decisiones deliberativas para experimentos del mundo real y decisiones de sostenibilidad (con respecto a la deliberación, véase, por ejemplo, Kowarsch et al., 2016).

El campo de estudios del futuro ofrece varios enfoques y métodos que apoyan la investigación sobre la sostenibilidad transformadora. En primer lugar, la generación de conocimiento de objetivos y de la transformación puede fomentarse mediante enfoques exploratorios (por ejemplo, a través de situaciones hipotéticas) y normativos (como el backcasting). En segundo lugar, los enfoques que se centran en las metodologías para la configuración de procesos de cambio, como el modelo de acción de futuros (Ramos, 2017) y la prospectiva experiencial, pueden proporcionar ideas para experimentos e intervenciones en el mundo real que tengan como objetivo hacer posible o lograr otros futuros; a su vez, tienen fuertes conexiones con los formatos participativos y la investigación-acción. En tercer lugar, los enfoques del área de la literacidad de futuros (Futures Literacy) apoyan la formación de la competencia anticipatoria, es decir, la capacidad de concebir los futuros como moldeables, comprender la influencia que las concepciones del futuro tienen en el presente y participar activamente en el trabajo con el futuro.

\section{Competencias transformadoras para promover el desarrollo sostenible}

La educación para el desarrollo sostenible (EDS) tiene como objetivo promover el desarrollo sostenible en la sociedad y desempeña un papel fundamental en los programas internacionales, al tiempo que llama a la acción; de los más reciente está "Educación para el Desarrollo Sostenible: Hacia el logro de los ODS” (EDs para 2030) (Unesco, 2019).

Uno de los aspectos más importantes de la EDs es formar a los estudiantes en competencias clave que les permitan contribuir al desarrollo sostenible como agentes de cambio. En una revisión de los enfoques de enseńanza y aprendizaje en la educación académica sobre la sostenibilidad, Wiek et al. (2011) identificaron cinco competencias clave que permiten a los estudiantes comprender y dar forma a los procesos de sostenibilidad y transformación. Éstas son: 1) competencia estratégica, 2) competencia de pensamiento sistémico, 3) competencia anticipatoria, 4) competencia normativa y 5) competencia interpersonal.

Las competencias clave fueron la base para diseñar los componentes educativos del LIT que se presentan en los siguientes párrafos.

\section{El Laboratorio de Innovación Transformadora (LIT)}

El formato del Laboratorio de Innovación Transformativa (LIT) tiene como objetivo integrar el enfoque del laboratorio transformador y transdisciplinario del mundo real en la educación superior. Los laboratorios operan en la intersección entre el modo de innovación experimental y el aprendizaje transformador para el desarrollo sostenible. El objetivo es crear espacios de aprendizaje del mundo real, integrados en los planes de estudio existentes, que permitan el análisis de los procesos de cambio desde una perspectiva de

64 Creación de espacios de aprendizaje y enseñanza para la investigación transformadora y trasdisciplinar... Matthias Wanner, Philip Bernert, Nele Fischer, Martina Schmitt. DIDAC 78 (2021): 60-71 
transformación sistémica, así como la promoción del desarrollo de competencias clave para el diseño estratégico y la implementación de estos procesos de cambio a través de la experimentación.

El núcleo del concepto del Lit es la integración del conocimiento teórico/metodológico, la experiencia y la reflexión: en un LIT, los estudiantes aprenden sobre las áreas temáticas de sostenibilidad e investigación transformadora y se les alienta a abordar específicamente los conocimientos de sistemas, de objetivos y de la transformación en el transcurso de un proyecto seleccionado por ellos mismos. Esta integración se realiza mediante la exploración de las perspectivas metodológicas de la investigación transformadora y transdisciplinaria, así como de la investigación de futuros. El diseño y la realización de un proyecto propio, tutelado, de investigación transformadora (a pequeña escala) ofrece a los estudiantes la oportunidad de aplicar la teoría y adquirir experiencia práctica. Este intercambio entre la teoría y la práctica no sólo mejora la experiencia de aprendizaje de los estudiantes, sino que les permite tener una visión crítica de sus propias capacidades y habilidades y de la influencia transformadora de sus propias acciones.

\section{Diseño y proceso del Laboratorio de Innovación Transformadora (LIT): investigación transformadora dentro de los formatos educativos existentes}

En lugar de desarrollar nuevos programas y cualificaciones académicas, el LIT está diseñado para integrar la investigación transformadora en el entorno de aprendizaje de los programas de estudio existentes. Los estudiantes trabajan de manera individual o en pequeños equipos y son asesorados a cada paso del camino por sus compañeros y el equipo docente. El LIT también alienta a los estudiantes a utilizar su proyecto de investigación como una tesis de maestría experimental. El diseño y el proceso del LiT funcionan, por tanto, dentro de la estructura semestral y se centran en la fase de investigación del laboratorio del mundo real (véase Figura 2).

\section{Figura 2}

Esquema integrado de un LIT ideal-típico.

Las fases individuales del LIT se muestran en los círculos coloreados del centro. En los recuadros de color gris se muestran los formatos de los eventos tal y como suceden a lo largo del proyecto. En cuanto a la metodología, las fases de la investigación transformadora se perfilan en la barra superior, mientras que la barra inferior muestra la secuencia cronológica del curso académico.

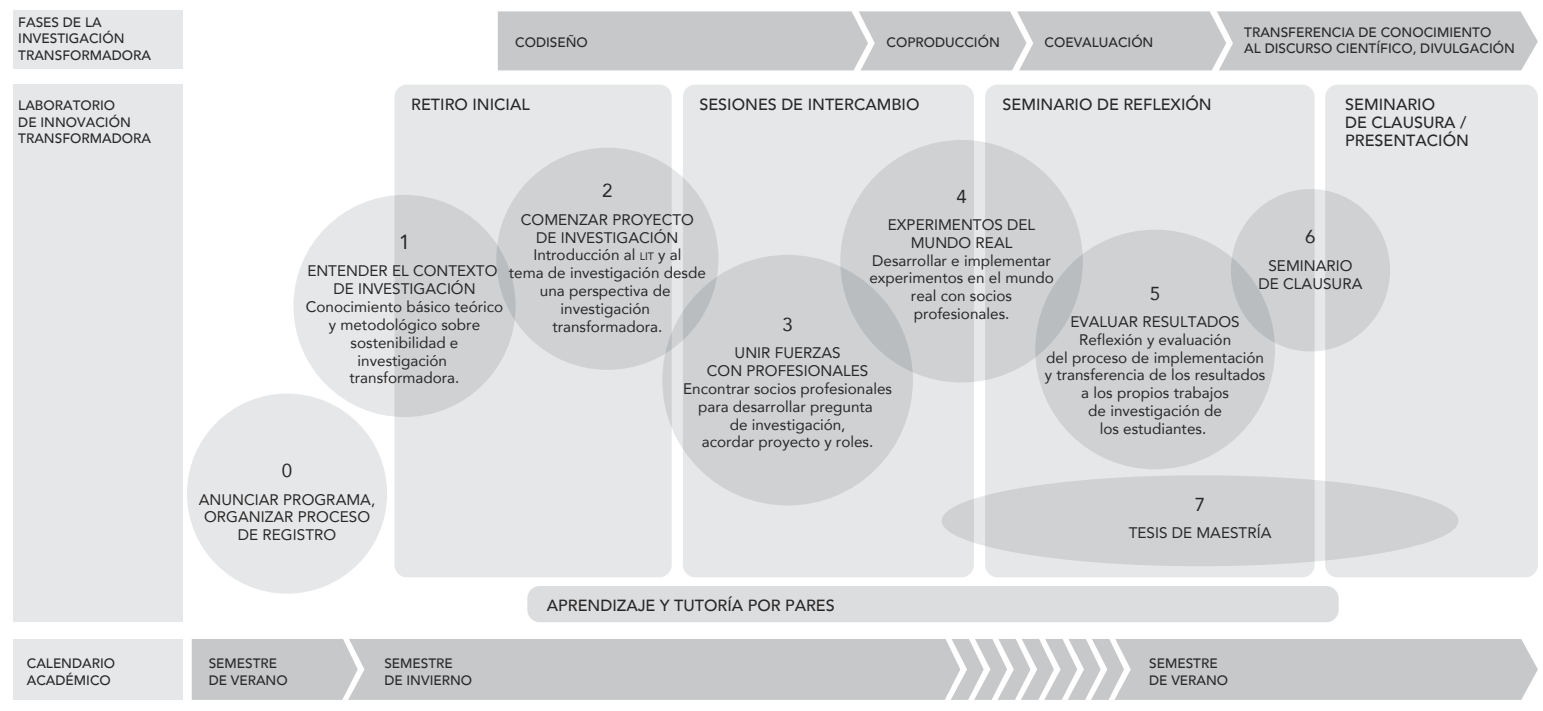

Adaptado de: "Transformative Innovation Lab: Handbook to Facilitate Students' Real-World Laboratory Projects to Promote Transformative and Transdisciplinary Competencies", por M. Wanner, M. Schmitt, N. Fischer \& P. Bernert, 2020, Wuppertal Institute for Climate, Environment and Energy, pp. 34-35 
El LIT está diseñado para implementarse en los dos últimos semestres de un programa de maestría. No obstante, la duración de un LIT puede adaptarse a los conocimientos previos de los estudiantes, a su participación en cualquier investigación existente y, especialmente, a la complejidad de los proyectos previstos y a su intención de utilizarlos en una tesis de maestría.

El primer semestre del LIT se enfoca en la enseñanza de conocimientos básicos, teóricos y metodológicos. Los estudiantes inician un proyecto de investigación y paralelamente lo codiseñan con los especialistas. Dependiendo del plan de estudios y de los requisitos de evaluación, los resultados de esta fase pueden tomar la forma de un informe de proyecto, un portafolio o una exposición para una tesis de maestría. El segundo semestre se centra en la implementación (coproducción) y la coevaluación, por ejemplo, como parte de una tesis de maestría. Al final del segundo semestre, los estudiantes concluyen el LIT con la realización de su proyecto.

\section{Formatos de Laboratorios de Innovación}

Transformadora (LIT): dar a los agentes de cambio las habilidades y competencias que necesitan

Al llevar a cabo experimentos del mundo real en colaboración con los especialistas, los estudiantes contribuyen directamente al inicio de los procesos de cambio en la sociedad y promueven la puesta en práctica de los conocimientos sobre el desarrollo sostenible. En lugar de percibirlos como competidores, los otros actores involucrados ven a los estudiantes como actores desafiantes y dinámicos (Larsson \& Holmberg, 2018). Esto permite crear una esfera de confianza que allana el camino para la innovación en materia de sostenibilidad.

Un LIT implica varios formatos para combinar 1) la reflexión sobre las habilidades, la motivación y las emociones individuales, 2) la adquisición de los conocimientos metodológicos y teóricos necesarios para llevar a cabo un proyecto de investigación y 3) el ámbito de implementación y experimentación.

Los formatos elegidos ofrecen una configuración parcialmente virtual y parcialmente del mundo real y una interacción fructífera entre las fases sincrónica y asíncrona del proceso de enseñanza y aprendizaje. Esto se produce en una mezcla de clases/seminarios (de educadores y profesionales invitados), salidas de campo (por ejemplo, visitas a laboratorios actuales del mundo real), tiempo para los propios proyectos de los estudiantes, ejercicios guiados, sesiones de reflexión e informes de experiencias de los estudiantes. Los formatos grupales desempeñan un papel importante para crear un espacio de confianza en el que experimentar, reflexionar y aprender, así como fomentar el intercambio y, cuando proceda, formar equipos para trabajar en proyectos de investigación.

Componentes clave del Laboratorio de Innovación Transformadora (LIT):

- Un retiro inicial de varios días en un lugar de aprendizaje no universitario marca el inicio del viaje.

- A lo largo del programa uit se llevan a cabo sesiones de intercambio sobre temas adaptados a la etapa en la que se encuentran los estudiantes tanto en formato presencial como en línea.

- El seminario de reflexión en la transición del diseño a la implementación tiene como objetivo presentar y cuestionar críticamente el proceso de aprendizaje individual sobre la manera de convertirse en un agente de cambio de la sostenibilidad.

- El seminario de clausura marca la conclusión del programa LIT con una celebración conjunta en la que los graduados presentan (públicamente) sus proyectos de investigación, idealmente con sus especialistas. Esto también ofrece la oportunidad de compartir experiencias tanto entre estudiantes y egresados del LIT como entre especialistas pioneros y responsables de la política pública.

Además de estos eventos, los estudiantes reciben tutorías continuas a lo largo del programa LIT. Éstas incluyen: 
- Aprendizaje entre pares. Durante el evento inaugural, los estudiantes forman equipos/tándems para apoyarse mutuamente de manera autoorganizada durante la realización del laboratorio, así como para aprender unos de otros a través del intercambio mutuo. El intercambio regular y la retroalimentación mutua con el grupo en su conjunto es un componente integral del laboratorio y es posible mediante el uso de canales de comunicación en línea y aplicaciones de mensajería instantánea.

- Coloquios. La realización periódica de estos eventos ofrece a los estudiantes una plataforma para presentar sus temas y el progreso realizado en sus proyectos de investigación o tesis de maestría, y para resolver temas tanto específicos como formales.

- Además, se ofrecen formatos de tutorías individuales durante el curso del laboratorio para proporcionar asesoría a los proyectos de investigación y a las tesis de maestría y fomentar el intercambio interdisciplinario. Lo ideal es que estos formatos ya estén previstos en los módulos del programa de estudios en los que se va a integrar un LIT.

- En el manual del uit (Wanner et al., 2020), disponible para su descarga, se ofrece información didáctica detallada sobre cómo llevar a cabo cada paso, con especial atención a la comprensión del contexto de investigación, los objetivos de aprendizaje y las opciones de implementación académica. La página web del proyecto ofrece listas de materiales y más sugerencias. ${ }^{2}$

\section{Aprendizajes del pilotaje del Laboratorio de Innovación Transformadora (LIT)}

Para poner a prueba el LIT, el modelo se piloteó en dos universidades alemanas en dos grupos entre 2018 y 2020. A través de esta aplicación fue posible perfeccionar el formato y enfrentarse a sus desafíos. A continuación, presentamos los resultados de la experiencia práctica obtenida al implementar y completar el LIT. También se pueden encontrar más detalles sobre los aprendizajes en el manual.

\section{¿Cómo se puede integrar un LIT en un plan} de estudios?

Selección del plan de estudios. Idealmente, los planes de estudio adecuados para un LIT deberían relacionarse o tener listos los objetivos del desarrollo sostenible, las opciones para su implementación y el papel que juega la investigación en los procesos de cambio. Es esencial que los estudiantes tengan un repertorio básico de metodologías de investigación que puedan utilizar en sus proyectos. Para calificar la tesis de maestría y los componentes evaluables, se requiere que los docentes tengan un conocimiento básico de los enfoques experimentales de este género.

\section{Implementación de un LIT en los módulos adecua-} dos de un plan de estudios actual. Los módulos existentes de un programa de estudios deben revisarse en términos de contenido, estructura y formatos de evaluación para encontrar un espacio adecuado para integrar el LIT. Los seminarios basados en proyectos, los formatos prácticos y los programas de maestría pueden conjugarse fácilmente con un LIT. Lo ideal es que la duración del módulo subyacente cubra dos semestres. El LiT puede conjugarse fácilmente con una amplia gama de requisitos de evaluación, especialmente con presentaciones, ensayos, portafolios, informes de prácticas y evaluaciones orales. La evaluación debe considerar la experiencia de aprendizaje abierto y el enfoque práctico del proyecto. Por lo tanto, los exámenes escritos son menos adecuados para evaluar un LIT.

El LIT debe planearse con antelación. Es recomendable empezar a planificar con anticipación (al menos con un semestre y medio) porque las universidades varían mucho en términos de flexibilidad con respecto a los requisitos formales e informales de los programas.

\section{Tomar en cuenta calendarios y horarios semestra-}

les. El LiT tiene una duración de dos semestres, por lo cual es importante garantizar que no se interrumpa el proceso de laboratorio típico-ideal ni se impida 
que el programa de estudios se complete dentro del periodo estándar de estudio.

Determinar un tamańo de grupo manejable para la participación en el LIT. Un LIT requiere tutorías intensivas de estudiantes. Por lo tanto, el máximo recomendado de estudiantes por docente es de $10 \mathrm{a}$ 15. Si se elige una tesis de maestría como resultado final, el grupo debe ser más pequeño.

Encontrar contactos y colaboradores adecuados, y unirse a varias redes. Puede resultar útil investigar quién, dentro de la oficina del rector, puede apoyar en el diseño de un seminario y un módulo para el LIT. La oficina de evaluaciones también es un punto de contacto importante en lo que respecta a la normativa de evaluación. Los proyectos de investigación universitarios en los que participan especialistas y partes interesadas locales proporcionan una buena infraestructura que no debe desaprovecharse. De este modo se pueden utilizar recursos adicionales, $\mathrm{y}$ los proyectos prácticos pueden ser apoyados y evaluados por la universidad.

Publicidad del programa LIT y su proceso de selección. Para reclutar a estudiantes interesados y motivados, resultó útil aplicar un proceso de solicitud que les permite presentar una carta de motivación (informal). Esto simplifica la planificación del laboratorio ya que permite evaluar los intereses de los estudiantes, sus conocimientos previos y sus expectativas.

\section{Consejos y recomendaciones para la}

aplicación del LIT: conclusiones de las

sesiones

Crear una base común de conocimientos. $\mathrm{Al}$ principio del laboratorio son esenciales las aportaciones teóricas sobre la investigación transformadora de la sostenibilidad y sobre el curso ideal-típico de un laboratorio del mundo real. Se debe invitar a expertos, tanto investigadores como especialistas. Aunque tome mucho tiempo, es importante que los estudiantes desarrollen una concepción común de los términos y metodologías especializadas del tema. Además de servir como fuentes de inspiración, las salidas de campo ayudan a recopilar ideas para proyectos.

Aprendizaje entre pares para un mejor aprovechamiento. Es aconsejable que los equipos/tándems de aprendizaje se formen de manera voluntaria y que se permita cambiar las constelaciones de los equipos como parte del proceso.

Diseño de la sesión: variedad y flexibilidad. Es importante establecer una combinación de clases/ seminarios, experiencias personales, tiempo para la reflexión y también para la diversión, actividades conjuntas y grupales (por ejemplo, calentamientos, eventos nocturnos autoorganizados, deportes y sesiones de meditación). También hay que dar cabida a enfoques alternativos, como caminatas de diálogo y espacios de reflexión y generación de ideas. Los juegos, como la simulación "El futuro del mundo", ${ }^{3}$ permiten abordar temas abstractos y ayudan a ilustrarlos. Los enfoques orientados a la acción, como una sesión rápida de seguimiento del proyecto, ayudan a los estudiantes a transferir los procesos ideales a su labor del proyecto. Las sesiones de conversación corta son otra forma sencilla para que los estudiantes compartan el estado actual de su progreso.

Comprender los roles y la ética de la investigación. Resulta esencial reflexionar sobre el propio papel en el proceso de investigación (transformadora) así como de las dimensiones éticas de las propias actividades (de investigación), y esto requiere proveer espacios adecuados para ello. Muchos participantes señalan que es difícil evitar caer en un papel unilateral de prestación de servicios, sin ningún interés personal por la investigación, o en el tradicional papel de analista "objetivo" o de activista.

\section{Desarrollo personal y gestión de las expectativas.} El proceso de investigación transformadora conlleva retos que inducen estrés al trabajar con los distintos actores implicados. Las altas expectativas contrastan con las intervenciones factibles. El establecimiento realista de metas es clave para el LIт. Hay que alentar

68 - Creación de espacios de aprendizaje y enseñanza para la investigación transformadora y trasdisciplinar... Matthias Wanner, Philip Bernert, Nele Fischer, Martina Schmitt. DIDAC 78 (2021): 60-71 
a los estudiantes a pensar más en pequeño que en grande para ayudarlos a afrontar la incertidumbre. Un ambiente de confianza dentro del grupo estimula a los estudiantes a compartir ideas. Se ha comprobado que es muy útil proporcionar información y ejercicios individuales sobre técnicas de trabajo emocional.

\section{Discusión y perspectivas}

Con este artículo pretendemos realizar una contribución útil y eficaz para incorporar la enseñanza de la investigación transdisciplinaria y transformadora con mayor firmeza en la educación superior. El logro de los objetivos de desarrollo sostenible —evitando el solucionismo, la exageración y las soluciones rápidas - exige formatos de investigación aplicada reflexivos y responsables. Al ser su aplicación exigente desde el punto de vista metodológico y ético, es aún más importante ofrecer tutorías prudentes y amplias para los procesos de aprendizaje.

Como todo proyecto, el LIT ha estado sujeto a limitaciones y han surgido una serie de cuestiones que deben tratarse con más detalle en el futuro. Entre ellas se encuentran, por ejemplo, la integración coherente en más y diferentes programas de estudio y la medición empíricamente sólida del desarrollo de las habilidades de los estudiantes a lo largo del LIT. También es importante analizar los factores de éxito y los obstáculos a la colaboración entre investigador y especialista, así como obtener una visión más diferenciada de sus distintas funciones. De igual manera, es incipiente la evaluación retrospectiva de cómo estos laboratorios del mundo real repercuten y afectan al desarrollo personal, al debate científico y a la promoción de prácticas sostenibles. Los formatos para la investigación y la enseñanza transdisciplinaria y transformadora deben demostrar su valor en estos tres aspectos.

\section{Agradecimientos}

Agradecemos a Elisa Schmelkes por su atenta traducción. Agradecemos a Willington Ortiz, como experto en transdisciplina de habla hispana, por su excelente trabajo sobre la coherencia de nuestros pensamientos y argumentos. Finalmente agradecemos a Montserrat Aranda por la minuciosa corrección de pruebas y el control lingüístico.

REFERENCIAS

Bergmann, M., Schäpke, N., Marg, O., Stelzer, F., Lang, D. J., Bossert, M., Gantert, M., Häußler, E., Marquardt, E., Piontek, F. M., Potthast, T., Rhodius, R., Rudolph, M., Ruddat, M., Seebacher, A. \& Sußmann, N. (2021). Transdisciplinary Sustainability Research in Real-World Labs: Success Factors and Methods for Change. Sustainability Science. Recuperado de https://doi.org/10.1007/s11625020-00886-8

Berkhout, F., Verbong, G., Wieczorek, A. J., Raven, R., Lebel, L. \& Bai, X. (2010). Sustainability Experiments in Asia: Innovations Shaping Alternative Development Pathways? Environmental Science \& Policy, 13(4), 261-271. Recuperado de https://doi.org/10.1016/j.envsci.2010.03.010

Bulkeley, H., \& Castán Broto, V. (2013). Government by Experiment? Global Cities and the Governing of Climate Change. Transactions of the Institute of British Geographers, 38(3), 361-375. Recuperado de https://doi.org/10.1111/j.1475-5661.2012.00535.x

Fuenfschilling, L., Frantzeskaki, N. \& Coenen, L. (2019). Urban Experimentation \& Sustainability Transitions. European Planning Studies, 27(2), 219-228. Recuperado de https://doi.org/10.1080/09654313.2018.1532977

Gibbons, M., Limoges, C., Nowotny, H., Schwartzman, S., Peter, S. \& Trow, M. (1994). The New Production of
Knowledge: The Dynamics of Science and Research in Contemporary Societies. Londres: Sage.

Grunwald, A. (2014). Modes of Orientation Provided by Futures Studies: Making Sense of Diversity and Divergence. European Journal of Futures Research, 2(1), 30.

Hirsch Hadorn, G., Bradley, D., Pohl, C., Rist, S. \& Wiesmann, U. (2006). Implications of Transdisciplinarity for Sustainability Research. Ecological Economics, 60(1), 119-128. Recuperado de https://doi.org/10.1016/j.ecolecon.2005.12.002

Hirsch Hadorn, G., Jäger, J. \& Akademien der Wissenschaften Schweiz. (2008). Handbook of transdisciplinary research. Alemania: Springer.

Kowarsch, M., Garard, J., Riousset, P., Lenzi, D., Dorsch, M. J., Knopf, B., Harrs, J.-A. \& Edenhofer, O. (2016). Scientific Assessments to Facilitate Deliberative Policy Learning. Palgrave Communications, 2(1). Recuperado de https://doi.org/10.1057/palcomms.2016.92

Lang, D. J., Wiek, A., Bergmann, M., Stauffacher, M., Martens, P., Moll, P., Swilling, M. \& Thomas, C. J. (2012). Transdisciplinary Research in Sustainability Science: Practice, Principles, and Challenges. Sustainability Science, 7(S1), 25-43. Recuperado de https://doi.org/10.1007/s11625-011-0149-x

Larsson, J. \& Holmberg, J. (2018). Learning While Creating Value for Sustainability Transitions: The Case of Challenge 
Lab at Chalmers University of Technology. Journal of Cleaner Production, 172, 4411-4420. Recuperado de https://doi.org/10.1016/j.jclepro.2017.03.072

Leicht, A., Heiss, J., Byun, W. J., \& Unesco. (2018). Issues and Trends in Education for Sustainable Development. Unesco. Recuperado de https://unesdoc.unesco.org/ark:/48223/ pf0000261445

Nevens, F., Frantzeskaki, N., Gorissen, L. \& Loorbach, D. (2013). Urban Transition Labs: Co-Creating Transformative Action for Sustainable Cities. Journal of Cleaner Production, 50, 111-122. Recuperado de https://doi.org/10.1016/j.jclepro.2012.12.001

Nowotny, H., Scott, P. \& Gibbons, M. (2001). Re-Thinking Science: Knowledge and the Public in an Age of Uncertainty. Cambridge: Polity Press.

Ramos, J. (2017). Linking Foresight and Action: Toward a Futures Action Research. En The Palgrave International Handbook of Action Research (S. 823-842). Alemania: Springer.

Schäpke, N., Stelzer, F., Caniglia, G., Bergmann, M., Wanner, M., Singer-Brodowski, M., Loorbach, D., Olsson, P., Baedeker, C. \& Lang, D. J. (2018). Jointly Experimenting for Transformation? Shaping Real-World Laboratories by Comparing Them. GAIA. Ecological Perspectives for Science and Society, 27(S1), 85-96. Recuperado de https://doi.org/10.14512/gaia.27.S1.16

Schneidewind, U., Singer-Brodowski, M., Augenstein, K. \& Stelzer, F. (2016). Pledge for a Transformative Science: A Conceptual Framework. Alemania: Wuppertal Papers. Recuperado de http://nbn-resolving.de/urn:nbn:de:bsz:wup4-opus-64142 Scholz, R. W. \& Steiner, G. (2015). The Real Type and Ideal Type of Transdisciplinary Processes: Part I - Theoretical Foundations. Sustainability Science, 10(4), 527-544. Recuperado de https://doi.org/10.1007/s11625-015-0326-4

Steffen, W., Broadgate, W., Deutsch, L., Gaffney, O. \& Ludwig, C. (2015). The Trajectory of the Anthropocene: The Great Acceleration. The Anthropocene Review, 2(1), 81-98. Recuperado de https://doi.org/10.1177/205301961456-4785

Steffen, W., Richardson, K., Rockström, J., Cornell, S. E., Fetzer, I., Bennett, E. M., Biggs, R., Carpenter, S. R., Vries, W. de, Wit, C. A. de, Folke, C., Gerten, D., Heinke, J., Mace, G. M., Persson, L. M., Ramanathan, V., Reyers, B. \& Sörlin, S. (2015). Planetary Boundaries: Guiding Human Development on a Changing Planet. Science, 347(6223). Recuperado de https://doi.org/10.1126/science.1259855
Thompson Klein, J. (2004). Prospects for Transdisciplinarity. Futures, 36(4), 515-526. Recuperado de https://doi.org/10.1016/j.futures.2003.10.007

Unesco - United Nations Educational, Scientific and Cultural Organization. (2019). Framework for the implementation of Education for Sustainable Development (ESD) beyond 2019 (S. 19). Recuperado de https://www.bne-portal.de/files/40\% 20C\%2023\%20ESD.pdf

United Nations (UN). (2015a). Nagoya Declaration in Higher Education for Sustainable Development. Recuperado de https:// sustainabledevelopment.un.org/content/documents/ 5864Declaration \% 20-\%20Higher\%20Education $\% 20$ for $\% 20$ Sustainable $\% 20$ Development $\% 20$ Nagoya $\% 20$ 2014.pdf

United Nations (UN). (2015b). Resolution adopted by the General Assembly on 25 September 2015 (A/RES/70/1.; Transforming our world: the 2030 Agenda for Sustainable Development). United Nations. Recuperado de https://undocs.org/A/RES/70/1

United Nations (UN). (2015c). The Millennium Development Goals Report 2015. United Nations. Recuperado de https:// www.un.org/millenniumgoals/2015_MDG_Report/pdf/ MDG\%202015\%20rev\%20(July\%201).pdf

Wanner, M., Hilger, A., Westerkowski, J., Rose, M., Stelzer, F. \& Schäpke, N. (2018). Towards a Cyclical Concept of Realworld Laboratories: A Transdisciplinary Research Practice for Sustainability Transitions. disP - The Planning Review, 54(2), 94-114. Recuperado de https://doi.org/10.1080/02513625.2018.1487651

Wanner, M., Schmitt, M., Fischer, N. \& Bernert, P. (2020). Transformative Innovation Lab: Handbook to Facilitate Students' Real-World Laboratory Projects to Promote Transformative and Transdisciplinary Competencies. Alemania: Wuppertal Institute for Climate, Environment and Energy. Recuperado de https://epub.wupperinst.org/frontdoor/index/index/docId/7685

wBGU-German Advisory Council on Global Change. (2011). World in Transition - A Social Contract for Sustainability. Wissenschaftlicher Beirat der Bundesregierung Globale Umweltveränderungen. Recuperado de https://www.wbgu.de/en/publications/publication/ world-in-transition-a-social-contract-for-sustainability

Wiek, A., Withycombe, L. \& Redman, C. L. (2011). Key Competencies in Sustainability: A Reference Framework for Academic Program Development. Sustainability science, 6(2), 203-218.

70 - Creación de espacios de aprendizaje y enseñanza para la investigación transformadora y trasdisciplinar... Matthias Wanner, Philip Bernert, Nele Fischer, Martina Schmitt. DIDAC 78 (2021): 60-71 


\section{SEMBLANZAS}

Matthias Wanner. Psicólogo y científico social, trabaja en el Instituto Wuppertal. Su investigación se enfoca en prácticas sociales sostenibles en los ámbitos de desarrollo urbano, aprendizaje, educación, participación y arte en entornos de laboratorios del mundo real transdisciplinarios y transformadores.

Philip Bernert. Licenciado en Ciencias de la Sostenibilidad por la Universidad de Leuphana, en Luneburgo. Su investigación se centra en los laboratorios del mundo real y en la investigación transdisciplinar de la sostenibilidad. Es facilitador de proyectos transdisciplinarios sobre transformaciones sostenibles en economías locales.

Nele Fischer. Tiene formación en estudios de medios de comunicación y estudios del futuro. Actualmente trabaja como investigadora en el Laboratorio de Ética de la Technische

Universität Berlin y es profesora de enfoques críticos y participativos en Estudios del Futuro en la Freie Universität Berlin. También trabaja como freelancer para apoyar el cambio organizativo.

Martina Schmitt. Licenciada en Ciencias Sociales trabaja en el Instituto Wuppertal desde 2003. Sus principales áreas de investigación son: educación para el desarrollo sostenible, conceptos para el aprendizaje transformador y la elaboración de materiales de enseńanza y aprendizaje.

\footnotetext{
${ }^{1}$ Este artículo es una traduccion del artículo en inglés "Creating Learning and Teaching Spaces for Transformative and Transdisciplinary Research: the Transformative Innovation Lab” que se publica en este mismo número 78 de la revista DIDAC.

${ }^{2}$ ttps://transformative-innovation-lab.de/en

${ }^{3}$ https://worldsfuture.socialsimulations.org/
} 